\title{
Toy model for the mean-field theory of hard-sphere liquids
}

\author{
Giorgio Parisi \\ Dipartimento di Fisica, Sezione INFN, and Unità INFM, I Università di Roma "La Sapienza", \\ Piazzale Aldo Moro 2, I-00185 Roma, Italy \\ František Slaninat* \\ Institute of Physics, Academy of Sciences of the Czech Republic \\ Na Slovance 2, CZ-18221 Praha, Czech Republic
}

\begin{abstract}
We investigate a toy model of liquid, based on simplified HNC equations in very large spatial dimension $D$. The model does not exhibit a phase transition, but several regimes of the behavior when $D \rightarrow \infty$ can be observed in different intervals of the density.

PACS numbers: 61.20.Gy; 05.20.Jj
\end{abstract}

\section{INTRODUCTION}

The theory of classical liquids [1],2] received recently an important stimulus from the the theory of structural glasses 3 [11. In the pioneering series of papers by Kirkpatrick and Thirumalai 12,13] the possible connection of structural-glass transition with the spin-glass transition in $p$-spin models was put forward. The analogy was then developed e. g. in the problem of minimally correlated sequences, which was shown to possess a glassy behavior without quenched randomness 14,15.

However, it would be desirable to put this analogy further. One of the difficulties occurring in structural glasses, when compared to spin glasses, comes from the absence of any kind of analytically solvable mean-field version of the model. In the case of spin glass, the role is played by the fully-connected Ising model, which is solvable very easily. The disordered version is the well-known Sherrington-Kikpatrick model [16], whose understanding is now very close to be complete 117 .

On the contrary, no analytical solution of a "meanfield" liquid is known, as far as we know. It can even sound not very reasonable to speak about a mean-field liquid, because the relevant high-density phase is characterized by strong short-range correlations, which can hardly be replaced by an effective medium. So, the meaning of the mean field should be better specified. In our investigation, we will understand by mean-field the situation which occurs in very high dimension, $D \rightarrow \infty$. The purpose of the present work is to introduce a simple model of a liquid, which is analytically solvable in the limit of infinite dimension, at least in a certain welldefined range of densities.

\section{SIMPLIFIED HNC EQUATIONS}

We consider a liquid composed of hard spheres with the diameter 1 . There is only one independent state variable, which is the spatial density of particles $\rho$.
The configuration of the liquid is described by the radial pair distribution function $g(r)=h(r)+1$. In the hypernetted chain (HNC) approximation [18] we have a closed set of equations for the correlation function $h(r)$

$$
\begin{aligned}
& h(r)+1=\exp (W(r)-\beta U(r)) \\
& \hat{W}(p)=\frac{\rho \hat{h}^{2}(p)}{1+\rho \hat{h}(p)} .
\end{aligned}
$$

The potential is $U(r)=0$ for $r>1$ and $U(r)=\infty$ for $r<1$. These equations can be interpreted as conditions for minimization of the free energy functional [3]

$$
\begin{aligned}
& \mathcal{F}[h]= \\
& \rho^{2} \int \mathrm{d} r r^{D-1}((h(r)+1)(\ln (h(r)+1)-1+U(r))+1) \\
& +\frac{1}{(2 \pi)^{D}} \int \mathrm{d} p p^{D-1} L_{3}(\rho \hat{h}(p))
\end{aligned}
$$

with $L_{3}(x)=-\ln (1+x)+x-\frac{x^{2}}{2}$. The function $L_{3}(x)$ has the following behavior: $L_{3}(x) \rightarrow \infty$ for $x \rightarrow-1$ and $L_{3}(x) \simeq-x^{3} / 3$ for $x \ll 1$.

Our main approximation will consist in replacement the function $L_{3}(x)$ by $L_{\infty}(x)$, where $L_{\infty}=\infty$ for $x<-1$ and $L_{\infty}=0$ otherwise. The motivation for this approximation is that we suppose that the main efffect of $L_{3}(x)$ is to forbid the region, where $-\rho \hat{h}(p)>1$. Then, minimization of the free energy functional amounts to satisfying the conditions

$$
\begin{aligned}
\rho \hat{h}(p) & \geq-1 \\
h(r) & \geq-1 \\
h(r) & =-1 \text { for } r<1
\end{aligned}
$$

which are in fact the minimum physical requirements for any correlation function $h(r)$. In this sense we are building a "minimum" model of a liquid. In addition to the constraints (3) we require that the function $h(r)$ depends continuously on the density. The absence of a solution 
which would be continuous in density would be a signal of a phase transition. This is, however, not found in the present calculations.

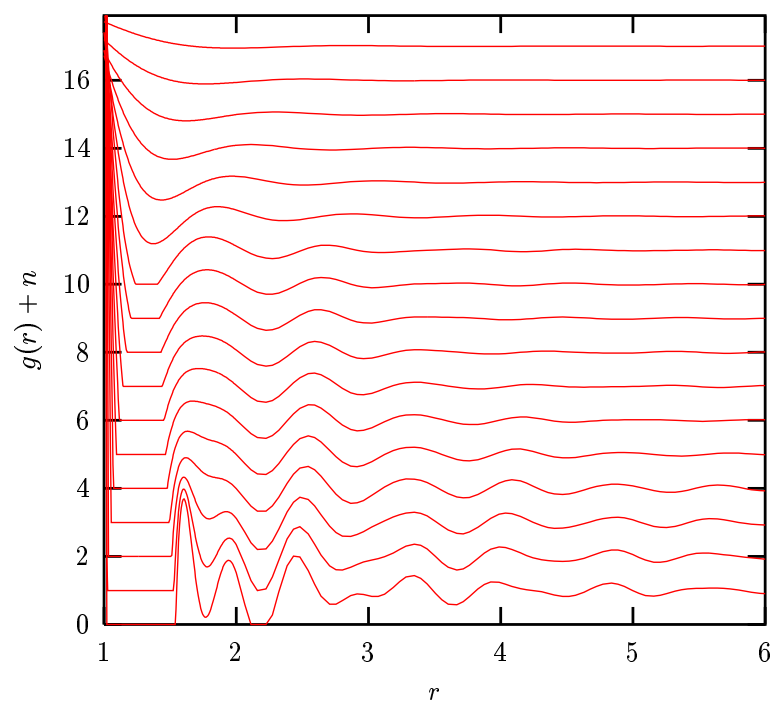

FIG. 1. Pair distribution function $g(r)=h(r)+1$ of the 3-dimensional model liquid, for densities (from top to bottom) $\rho=0.6,0.7,0.8,0.9,1.00,1.1,1.2,1.23,1.26,1.29,1.32,1.35$, $1.38,1.41,1.44,1.47,1.5$. The $n$-th curve from the bottom is shifted by $n$ upwards.

In $3 \mathrm{D}$ we can compute the function $h(r)$ numerically by increasing slowly the density $\rho$ and adjusting iteratively the function $h(r)$ so as the conditions (3) are satisfied. The resulting pair distribution function $g(r)=h(r)+1$ is shown in the Fig. 1. The Fourier transform $\hat{h}(p)$ for $\rho=1.2$ is shown in Fig. 2.

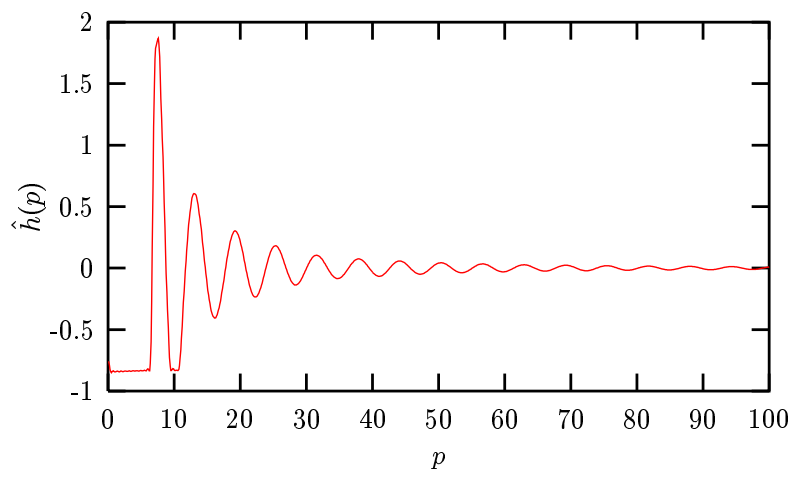

FIG. 2. Fourier transform of the correlation function for 3-dimensional liquid, at the density $\rho=1.2$.

We can see that for densities up to about $\rho=1$ the pair distribution function agrees qualitatively with the well-known results of HNC approximation or numerical simulations (see [1]). However, at about $\rho=1.2$ the behavior changes. A gap opens between the principal peak at $r=1$ and the secondary peak at $r \simeq 2$. the gap broadens with increased density and at about $\rho \simeq 1.5$ a second gap occurs around $r \simeq 2.2$. We observed, that further compression leads to the occurrence of a third gap separating the peaks at $r \simeq 1.6$ and $r \simeq 2$. We expect that continued increase of the density results in increased number of gaps.

The presence of the gaps is an artifact of the approximation. In reality the values of $g(r)$ will not be strictly zero, but small.

From the value of the radial distribution function at $r=1$ the pressure can be computed [1] and the resulting equation of state is shown in Fig. 3, together with the results obtained by solving the HNC equations (11) and the formula computed in the Percus-Yevick (PY) approximation [1]. We can see that our model behaves qualitatively in the same way as the other approximations, even though quantitative agreement is poor. On the other hand, the equation of state of our model does not differ from either HNC or PY approximation more than these two approximations differ one from the other.

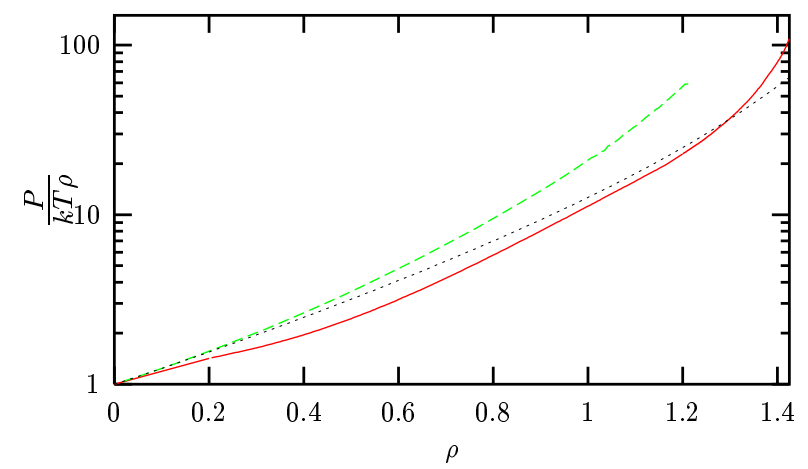

FIG. 3. Equation of state for 3-dimensional liquid. Our model: full line (red). HNC approximation: dashed line (green). Percus-Yevick approximation: dotted line (black).

We can see from these results, that the present approach in 3D gives at least qualitatively sensible results. However, our aim is not to provide a new approximation for real three-dimensional liquids, but a model which describes reasonably well the qualitative features of a liquid and is soluble in the limit of infinite spatial dimension. This will be done in the next section.

\section{SOLUTION OF THE MODEL IN HIGH DIMENSION}

In this section we will investigate a $D$-dimensional version of the model, with $D=2 N+3$ and $N \rightarrow \infty$. The main quantity of interest is again the correlation function $h(r)=g(r)-1$. We will suppose that it has the form $h(r)=h_{0}(r)+\bar{h}(r)$ where $h_{0}(r)=-\theta(1-r)$ and $\bar{h}(r)=0$ for $r<1$.

The pressure is directly related to the value of that $r=1$, more precisely to $\lim _{r \rightarrow 1^{+}} h(r)=\bar{h}\left(1^{+}\right)$(see [1]) 


$$
\frac{1}{k T} P=\rho+\frac{1}{2} V_{D} \rho^{2}\left(1+\bar{h}\left(1^{+}\right)\right) .
$$

In the following, we will use rescaled quantities. We will use $\bar{\rho}=\rho V_{D}$ for the density, while for the pressure we use $\bar{P}=\frac{V_{D} P}{k T}$.

In order to check the conditions (3) we should know the properties of the Fourier transform in high dimension. The relevant formulae used in this section are given in the Appendix A.

In the zeroth approximation $\hat{h}(\hat{p})=-V_{D} \Psi(\hat{p})$, which is correct as long as $\rho<1 / V_{D}$. So, for $\bar{\rho}<1$ we have $\bar{h}(r)=0$ and the pressure is given by first virial correction,

$$
\bar{P}=\bar{\rho}+\frac{1}{2} \bar{\rho}^{2} .
$$

For $\bar{\rho}>1$ we rewrite $h$ in the form

$$
h(r)=h_{0}^{*}(r)+\bar{h}^{*}(r)
$$

where still $\bar{h}^{*}=\bar{h}(r)$ for $r>1$, but $\bar{h}^{*}(r)$ is continuous for all $r$. The Fourier transform of $h_{0}^{*}$ is easy to compute, if we know the value $h^{*}\left(1^{-}\right)=-1-A$, where $A=\bar{h}\left(1^{+}\right)=$ $\hat{h}_{1}(1)$. Then

$$
\widehat{h_{0}^{*}}(\hat{p})=-(1+A) V_{D} \Psi_{0}(\hat{p})
$$

In order to ensure $\hat{h}(\hat{p}) \geq 1 / \rho$ everywhere, we set

$$
\widehat{h^{*}}(\hat{p})=\theta_{1}\left((A+1) V_{D} \Psi_{0}(\hat{p})-\frac{1}{\rho}\right) .
$$

(We denote $\theta_{1}(x)=x$ for $x>0, \theta_{1}(x)=0$ for $x \leq 0$.)

The relevant quantity is

$$
\rho_{1}=\frac{\ln \bar{\rho}}{N} .
$$

Indeed, one particle occupies space $2^{-D} V_{D}$, so an absolute upper bound for the density is $\rho_{1}<\ln 4$.

Let us suppose that $\hat{p}_{c}<1$. This condition restrict the range of densities investigated to a certain interval, which will be found in what follows.

For $\hat{p}<1$ the function $\Psi_{0}(\hat{p})=\exp \left(-N \phi_{0}(\hat{p})\right)$ is monotonously decreasing and we have the following equation for $\hat{p}_{c}$.

$$
\phi_{0}\left(\hat{p}_{c}\right)=\rho_{1}-\frac{\ln (A+1)}{N}
$$

When computing the inverse Fourier transform of $\hat{h}^{*}(\hat{p})$ we need only the behavior around the point $\hat{p}_{c}$, which is

$$
\hat{h}^{*}(\hat{p}) \simeq-V_{D} \Psi_{0}^{\prime}\left(\hat{p}_{c}\right) \hat{p}_{c}\left(1-\frac{\hat{p}}{\hat{p}_{c}}\right)
$$

and gives, using (37)

$$
\hat{h}_{1}(r)=\left(\frac{N}{2 \pi}\right) \frac{V_{D}^{2} \hat{p}_{c}^{D+1}\left(-\Psi_{0}^{\prime}\left(\hat{p}_{c}\right)\right)(A+1)}{D+1} \Psi_{0}\left(\hat{p}_{c} r\right)
$$

We obtain immediately the equation for $A$

$$
\left.A=\left(\frac{N}{2 \pi}\right)^{D} \frac{N}{D+1} V_{D}^{2} \hat{p}_{c}^{D+1} \phi_{0}^{\prime}\left(\hat{p}_{c}\right)\right)(A+1) \Psi_{0}^{2}\left(\hat{p}_{c}\right) .
$$

In the interval $\hat{p}_{c}<1$ we have

$$
\phi_{0}^{\prime}\left(\hat{p}_{c}\right)=\frac{\hat{p}_{c}}{1+\sqrt{1-\hat{p}_{c}^{2}}}<\hat{p}_{c} .
$$

Hence, if we suppose that a solution such that $A<1$ exists, we have

$$
A \leq\left(\frac{N}{2 \pi}\right)^{D} V_{D}^{2} \hat{p}_{c}^{D+2} \Psi_{0}^{2}\left(\hat{p}_{c}\right) \simeq \exp \left(-2 N K\left(\hat{p}_{c}\right)\right)
$$

where

$$
K(\hat{p})=\ln \left(1+\sqrt{1-\hat{p}^{2}}\right)-\sqrt{1-\hat{p}^{2}}-\ln \hat{p} .
$$

We have $K(1)=0$ and $K(\hat{p})=-\sqrt{1-\hat{p}^{2}} / \hat{p}<0$, so $K(\hat{p})>0$ for $\hat{p}<1$. For fixed $\hat{p}_{c}<1$ and $N \rightarrow \infty$ we have $A \ll 1$ and therefore we can neglect $A$ in the equations (10) and (13).

So, we can conclude that in the range of densities $\rho_{1}<$ $\rho_{1 c}=\phi_{0}(1)=1-\ln (2)=0.3068 \ldots$ the following equation for $\hat{p}_{c}$ holds

$$
\rho_{1}=\ln \left(1+\sqrt{1-\hat{p}_{c}^{2}}\right)-\sqrt{1-\hat{p}_{c}^{2}}+1-\ln 2 .
$$

The solution of the latter equation can be easily obtained in the form of power series. We show here only first several terms. The expansion up to order 16 is given in the Appendix B and the graph is shown in Fig. 1 .

$$
\hat{p}_{c}^{2}=4 \rho_{1}-2 \rho_{1}{ }^{2}-\frac{2}{3} \rho_{1}{ }^{3}-\frac{5}{6} \rho_{1}^{4}-O\left(\rho_{1}{ }^{5}\right) .
$$

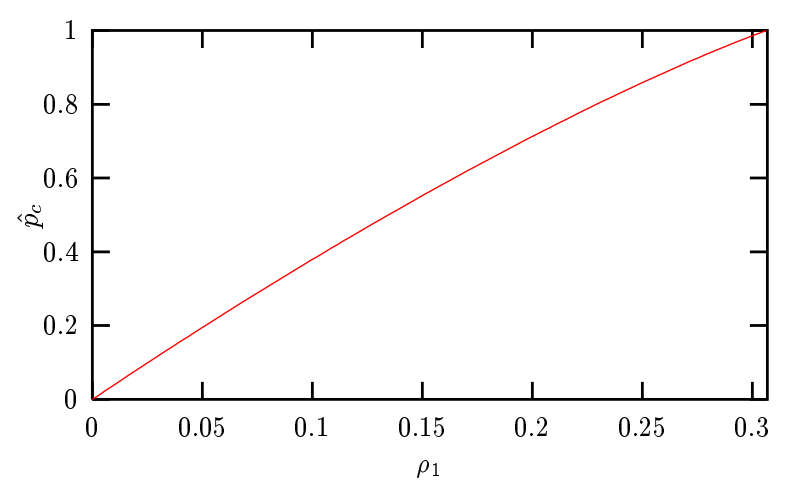

FIG. 4. Dependence of the momentum $\hat{p}_{c}$ on the rescaled density in the regime $\hat{p}_{c}<1$. 
From the solution Eq. (17) we can compute the pressure. We write $\bar{P}=\bar{\rho}+\frac{1}{2} \bar{\rho}^{2}+P_{c}$ and re-scale the correction as $P_{c}=\exp \left(N P_{1}\right)$. We obtain

$$
P_{1}=2-2 \ln 2+\ln \hat{p}_{c}^{2}
$$

We can see that the density $\rho_{1 t}=\phi_{0}\left(\frac{2}{\mathrm{e}}\right)=0.14676 \ldots$ separates two regimes. For $\rho_{1}<\rho_{1 t}$ the correction $P_{c}$ to the lowest virial becomes negligible for large $N$, while for $\rho_{1}>\rho_{1 t}$ the correction diverges for $N \rightarrow \infty$. The graph of the function $P_{1}\left(\rho_{1}\right)$ is shown in Fig. 5 .

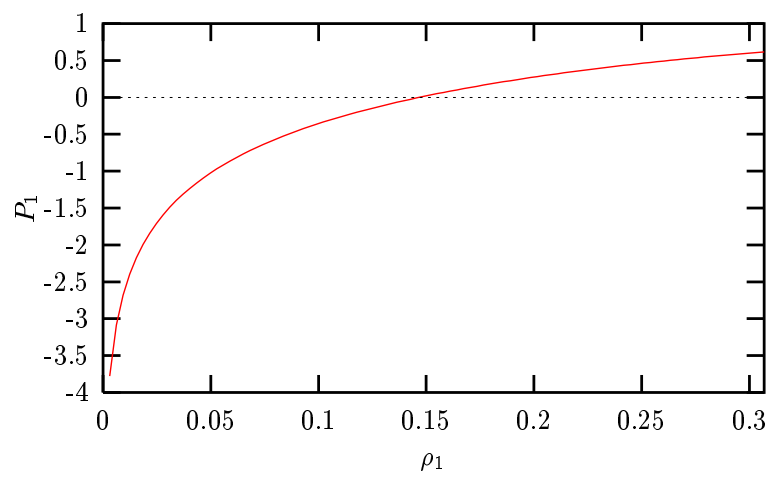

FIG. 5. Equation of state for our model in the limit of infinite dimension, in the range of densities where $\hat{p}_{c}<1$.

For the correlation function in the interval $r \hat{p}_{c}<1$ we have $h(r)=\exp \left(N h_{1}(r)\right)$, where

$$
\begin{aligned}
& h_{1}(r)= \\
& 1-2 \ln 2+\ln \hat{p}_{c}^{2}-\rho_{1}+ \\
& +\sqrt{1-\hat{p}_{c}^{2} r^{2}}-\ln \left(1+\sqrt{1-\hat{p}_{c}^{2} r^{2}}\right) .
\end{aligned}
$$

and for $r \hat{p}_{c}>1$ we can use the following scaling $\hat{h}(r)=$ $\exp \left(N h_{1}(r)\right) \cos \left(N h_{2}(r)\right)$ where, using the expressions (32) and (33), we obtain

$$
\begin{gathered}
h_{1}(r)=1-2 \ln 2+\ln \hat{p}_{c}-\rho_{1}-\ln r \\
h_{2}(r)=\sqrt{\hat{p}_{c}^{2} r^{2}-1}-\arctan \sqrt{\hat{p}_{c}^{2} r^{2}-1} .
\end{gathered}
$$

We can also see that $\mid h_{1}(r) \ll 1$ for $\rho_{1}<\rho_{1 c}$, so that $g(r)>0$ for $r>1$ and the gaps in $g(r)$, discussed in the last section do not occur. However, when $\rho_{1}$ approaches $\rho_{1 c}$ the absolute value of $h_{1}(r)$ can be of order $O(1)$ and a gap can appear at the density $\rho_{1 c}$. The detailed investigation of this process and the behavior of the model for $\rho_{1}>\rho_{1 c}$ remains an open question.

\section{CONCLUSIONS}

We investigated a simple model for a hard-sphere liquid. By numerical solution in 3 dimensions, we found a qualitatively realistic behavior. The results for the equation of state are compatible with the hypernetted chain and Percus-Yevick approximations.

We solved the model analytically in the limit of large spatial dimension. We found that two scales of density and pressure appear, which corresponds to two regimes of density. For $\bar{\rho}<1$ the equation of state is given by first virial correction (5), while for $\bar{\rho}>1$ the quantity relevant to the further virial corrections is $\rho_{1}=\ln \bar{\rho} / N$ and the pressure correction itself scale as $P_{1}=\ln P_{c} / N(19)$. We have found the solution in the interval $0<0 \rho_{1}<\rho_{1 c}=$ $0.3068 \ldots$. Two regimes are present within this interval. For $\rho_{1}<\rho_{1 t}=0.14676 \ldots$ the correction $P_{c}$ vanishes for large $N$, while for $\rho_{1}<\rho_{1 t}$ it diverges for large $N$.

It should be expected, that the presence of presence of gaps in $g(r)$ will lead to qualitatively different behavior for densities higher than $\rho_{1 c}$.

\section{ACKNOWLEDGMENTS}

One of us (F.S.) wishes to thank to the INFN section of Rome University "La Sapienza" for financial support and kind hospitality. This work was supported by the project No. 202/00/1187 of the Grant Agency of the Czech Republic.

\section{APPENDIX A}

Here we derive the formula for the Fourier transform in high dimension.

Fourier transform in $D=2 N+3$ dimensions is $(x$ and $k$ are $D$-dimensional vectors)

$$
\begin{gathered}
\hat{f}_{D}(k)=\int \mathrm{d}^{D} x \mathrm{e}^{\mathrm{i} x k} f_{D}(x) \\
f_{D}(x)=(2 \pi)^{-D} \int \mathrm{d}^{D} k \mathrm{e}^{-\mathrm{i} x k} \hat{f}_{D}(k) .
\end{gathered}
$$

We suppose that the functions depend only on the radial coordinate, $f(r)=f_{D}(x)$ for $r=|x|$ and $\hat{f}(p)=\hat{f}_{D}(k)$ for $p=|k|$. After rescaling $\hat{p}=p / N$ we will finally have

$$
\begin{aligned}
& \hat{f}(\hat{p})= \\
& C_{N} \int_{0}^{\infty} \mathrm{d} r \int_{-1}^{1} \mathrm{~d} z\left[r^{2\left(1+\frac{1}{N}\right)}\left(1-z^{2}\right) \mathrm{e}^{\mathrm{i} \hat{p} r z}\right]^{N} f(r)
\end{aligned}
$$

where the constant $C_{N}$ is fixed by condition that for $f(r)=\theta(1-r)$ we have $\hat{f}(0)=V_{D}$ with $V_{D}$ volume of the $D$-dimensional sphere,

$$
V_{D}=\frac{2 \pi^{D / 2}}{D \Gamma\left(\frac{D}{2}\right)} \simeq\left(\frac{\mathrm{e} \pi}{N}\right)^{N}
$$

Similarly for the inverse Fourier transform we will have 


$$
\begin{aligned}
& f(r)= \\
& \hat{C}_{N} \int_{0}^{\infty} \mathrm{d} \hat{p} \int_{-1}^{1} \mathrm{~d} z\left[\hat{p}^{2\left(1+\frac{1}{N}\right)}\left(1-z^{2}\right) \mathrm{e}^{\mathrm{i} \hat{p} r z}\right]^{N} \hat{f}(\hat{p})
\end{aligned}
$$

with coefficient

$$
\hat{C}_{N}=C_{N}\left(\frac{N}{2 \pi}\right)^{D}
$$

The calculation of the Fourier transform can be performed by the saddle-point method. The essential result is the Fourier transform of the surface of unit sphere, $f(r)=\delta(r-1)$. We obtain $\hat{f}(\hat{p}) \propto \Psi(\hat{p})$ where

$$
\Psi(\hat{p})=\Psi_{0}(\hat{p})=\exp \left(-N \phi_{0}(\hat{p})\right)
$$

for $\hat{p}<1$ and

$$
\Psi(\hat{p})=\Psi_{1}(\hat{p})=\exp \left(-N \phi_{1}(\hat{p})\right) \cos \left(N \phi_{2}(\hat{p})\right)
$$

for $\hat{p}>1$.

The explicit form of the functions $\phi_{0}, \phi_{1}, \phi_{2}$ is given by

$$
\begin{aligned}
& \phi_{0}(\hat{p})=1-\ln 2+\ln \left(1+\sqrt{1-\hat{p}^{2}}\right)-\sqrt{1-\hat{p}^{2}} \\
& \phi_{1}(\hat{p})=1-\ln 2+\ln \hat{p} \\
& \phi_{2}(\hat{p})=\sqrt{\hat{p}^{2}-1}-\arctan \sqrt{\hat{p}^{2}-1} .
\end{aligned}
$$

Note that $\Psi(0)=1$.

From here we can deduce the following Fourier transforms $(\theta(x)=1$ for $x>0$ and $\theta(x)=0$ for $x<0)$. For $f(r)=A \theta\left(r_{0}-r\right):$

$$
\hat{f}(\hat{p})=A V_{D} r_{0}^{D} \Psi\left(\hat{p} r_{0}\right) .
$$

For $f(r)=A\left(1-r / r_{0}\right) \theta\left(r_{0}-r\right)$ :

$$
\hat{f}(\hat{p})=\frac{A V_{D} r_{0}^{D}}{D+1} \Psi_{0}\left(\hat{p} r_{0}\right) .
$$

Because the inverse Fourier transform has the same form and differs only in the factor $\hat{C}_{N}$ instead of $C_{N}$, we can also immediately write for $\hat{f}(\hat{p})=A \theta\left(\hat{p}_{c}-\hat{p}\right)$ :

$$
f(r)=\left(\frac{N}{2 \pi}\right)^{D} A V_{D} \hat{p}_{c}^{D} \Psi\left(\hat{p}_{c} r\right)
$$

and for $\hat{f}(\hat{p})=A\left(1-\hat{p} / \hat{p}_{c}\right) \theta\left(\hat{p}_{c}-\hat{p}\right)$

$$
f(r)=\left(\frac{N}{2 \pi}\right)^{D} \frac{A V_{D} \hat{p}_{c}^{D}}{D+1} \Psi\left(\hat{p}_{c} r\right) .
$$

\section{APPENDIX B}

Using Maple V we get the following expansion for the solution of equation (17).

$$
\begin{aligned}
& \hat{p}_{c}^{2}= \\
& 4 \rho_{1}-2 \rho_{1}{ }^{2}-2 / 3 \rho_{1}{ }^{3}-5 / 6 \rho_{1}{ }^{4}-\frac{41}{30} \rho_{1}{ }^{5}-\frac{469}{180} \rho_{1}{ }^{6}- \\
& -\frac{6889}{1260} \rho_{1}{ }^{7}-\frac{24721}{2016} \rho_{1}{ }^{8}-\frac{2620169}{90720} \rho_{1}{ }^{9}-\frac{64074901}{907200} \rho_{1}{ }^{10}- \\
& -\frac{1775623081}{9979200} \rho_{1}{ }^{11}--\frac{1571135527}{3421440} \rho_{1}{ }^{12}- \\
& -\frac{1882140936521}{1556755200} \rho_{1}{ }^{13}-\frac{70552399533589}{21794572800} \rho_{1}{ }^{14}- \\
& -\frac{2874543652787689}{326918592000} \rho_{1}{ }^{15}-\frac{25296960472510609}{1046139494400} \rho_{1}{ }^{16} .
\end{aligned}
$$

* Electronic address: slanina@fzu.cz

[1] C. A. Croxton, Liquid state physics - A statictical mechanical introduction (Cambridge University Press, 1974).

[2] J. A. Barker and D. Henderson, Rev. Mod. Phys. 48, 587 (1976).

[3] M. Mézard and G. Parisi, J. Phys. A: Math. Gen. 29, 6515 (1996).

[4] M. Mézard and G. Parisi, Phys. Rev. Lett. 82, 747 (1999).

[5] M. Mézard and G. Parisi, J. Chem. Phys. 111, 1076 (1999).

[6] B. Coluzzi, M. Mézard, G. Parisi, and P. Verrocchio, J. Chem. Phys. 111, 9039 (1999).

[7] B. Coluzzi, G. Parisi, and P. Verrocchio, J. Chem. Phys. 112, 2933 (2000).

[8] M. Mézard, Physica A 256, 352 (1999).

[9] B. Doliwa and A. Heuer, cond-mat/9912421 (1999).

[10] B. Coluzzi, G. Parisi, and P. Verocchio, Phys. Rev. Lett. 84, 306 (2000).

[11] C. Dasgupta and O. T. Valls, cond-mat/0002081 (2000).

[12] T. R. Kirkpatrick and D. Thirumalai, Phys. Rev. Lett. 58, 2091 (1987).

[13] T. R. Kirkpatrick and D. Thirumalai, Phys. Rev. B 36, 5388 (1987).

[14] E. Marinari, G. Parisi and F. Ritort, J. Phys. A: Math. Gen. 27, 7615 (1994).

[15] E. Marinari, G. Parisi and F. Ritort, J. Phys. A: Math. Gen. 27, 7647 (1994).

[16] M. Mézard, G. Parisi, and M. A. Virasoro, Spin Glass Theory and Beyond (World Scientific, Singapore, 1987).

[17] A. P. Young (editor), Spin glasses and random fields (World Scientific, Singapore, 1997).

[18] E. Meeron, J. Math. Phys. 1, 192 (1960). 